\title{
Gastro-entérites en milieux des réfugiés au Tchad
}

\author{
Nadlaou BESSIMBAYE ${ }^{1,3 *}$, Adelsalam TIDJANI ${ }^{2}$, Khadidjia GAMOUGAME ${ }^{1}$, Brahim \\ BOY OTCHOM ${ }^{4}$, Guelmbaye NDOUTAMIA ${ }^{5}$, Lassana SANGARE ${ }^{7}$, Nicolas BARRO ${ }^{3}$, \\ et Alfred TRAORE ${ }^{3}$
}

\author{
${ }^{1}$ Hôpital Général de Référence Nationale de N'Djamena (HGRN), Tchad. \\ ${ }^{2}$ Faculté des Sciences de la Santé Humaine (FSSH), Université de N'Djamena, Tchad. \\ ${ }^{3}$ Centre de Recherche en Sciences Biologiques Alimentaires et Nutritionnelles (CRSBAN), \\ Université de Ouagadougou, Burkina Fasso. \\ ${ }^{4}$ Faculté des Sciences Exactes et Appliquées (FSEA), Université de N'Djamena, Tchad. \\ ${ }^{5}$ Laboratoire de Recherches Vétérinaires et Zootechniques (LRVZ) de N'Djamena, Tchad. \\ ${ }^{6}$ Unité de Formation et de Recherche en Sciences de la Santé (UFR/SDS), Université de Ouagadougou, \\ Centre Hospitalier Universitaire Yalgado Ouedraogo de Ouagadougou (CHU-YO), \\ Bessimbaye Nadlaou. \\ "Auteur correspondant, E-mail: nadlaobes@yahoo.fr; Tel : +23566 373647 / +23590209073
}

\section{RESUME}

Les réfugiés et déplacés de guerre sont des personnes vulnérables. C'est pourquoi, nous avons entrepris une étude des gastro-entérites dans leurs milieux au Sud et à l'Est du Tchad. Cette étude a concerné 53 patients dans les camps du Sud dont 28,30\% sont des enfants de 0 à 5 ans. Elle a été couplée d'une enquête sur l'état nutritionnel de 205 réfugiés dans les deux zones. L'isolement et l'identification des entéropathogènes dans les selles ont été réalisés au laboratoire de l'Hôpital Général de Référence Nationale (HGRN), en utilisant les milieux Hektoen, Mueller-Hinton, EMB, Saboraud Chloramphénicol et la galerie API ${ }^{\circledR} 20$ E. La microscopie, les tests de filamentation et biochimiques ont permis de caractériser et de déterminer les taux des infestations parasitaires, fongiques et d'infections bactériennes qui sont respectivement de $18,86 \%, 11,32 \%$ et $24,52 \%$. La caractérisation de la sensibilité des agents bactériens Salmonella, Shigella et Escherichia coli a montré une résistance de $85 \%$ aux bêta-lactamines. En revanche, $98 \%$ des germes sont sensibles aux fluoroquinolones. Cette étude nous a permis non seulement de connaître les caractéristiques des diarrhées liées à l'état nutritionnel des réfugiés, mais également, de mettre en évidence une antibiothérapie efficace permettant la mise en place d'un programme de prévention contre les maladies diarrhéiques et d'amélioration de leur état nutritionnel dans les milieux confinés. L'amélioration de l'état nutritionnel des enfants pourrait comporter :

la distribution régulière et complète des aliments riches en vitamines; l'extension des programmes d'alimentation d'appoint et le logement adéquat pour les réfugiés.

(C) 2013 International Formulae Group. All rights reserved.

Mots clés: Diarrhée, entéropathogènes, nutritionnel, antibiotique, HGRN.

\section{INTRODUCTION}

De 2003 à nos jours, le Tchad a accueilli environ 230000 réfugiés soudanais, 85000 centrafricains ayant fui respectivement les hostilités du Darfour (Est du Tchad), et de la République Centrafricaine (Sud du Tchad) et 180000 déplacés tchadiens (IMC, 2010). Il est établi 12 camps des immigrés le long de la 
frontière Tchado-soudanaise et 3 camps des réfugiés à Goré au Sud du Tchad. Face à ce nombre accru des réfugiés et déplacés, il se pose le problème de logement, de latrines et de l'approvisionnement en eau potable par les services de base des humanitaires, indispensables pour accueillir les réfugiés et les aider à survivre dans un environnement naturel hostile avec de maigres ressources locales. En effet, la vie dans les camps n'est pas facile dans un milieu désertique de l'Est du Tchad, où l'eau et le bois sont extrêmement rares. L'accès à l'eau potable et les soins demeurent un énorme problème. La précarité de la situation socio-économique et sanitaire des réfugiés était à l'origine des épidémies de choléra en République Démocratique du Congo (165 cas dont 25 décès), en Ouganda ( 25 cas dont 2 décès) et en Thaïlande (370 cas) (Breiman et al., 2009). $\mathrm{Au}$ Tchad, environ 19000 personnes notamment 15900 enfants de moins de 5 ans, meurent chaque année de diarrhée. Ces décès sont à presque $90 \%$ directement attribués à l'impureté de l'eau et au manque d'assainissement et hygiène (WSP, 2012).

La présente étude vise à établir la prévalence des entéropathogènes impliqués dans les maladies diarrhéiques liées à l'état nutritionnel des réfugiés, et également à déterminer la sensibilité des germes aux antibiotiques couramment utilisés dans les structures sanitaires en milieu des réfugiés au Tchad. Les résultats de ce travail pourront être un bon outil de sensibilisation en vue de définir une stratégie adéquate de prévention dans les milieux confinés.

\section{MATERIEL ET METHODES}

\section{Cadre, période et type de l'enquête}

Il s'agit des enquêtes prospectives qui ont été effectuées du 17 au 19 décembre 2010 sur les conditions de vie des réfugiés dans le camp de GozAmir à l'Est du Tchad, et du 20 au 24 avril 2011 dans les camps des réfugiés à Goré au Sud, sur les gastro-entérites liées à l'état nutritionnel des réfugiés.

\section{Critère d'inclusion}

Tout réfugié souffrant de troubles digestifs, repéré dans le ménage au camp ou au centre de santé fait l'objet de l'étude.

Chaque réfugié inclus dans l'étude a fait l'objet de prélèvement des selles dans un flacon stérile. Deux fiches de collecte des données d'investigation (Fiche de coproculture et une fiche de collecte de données sur les conditions de vie) ont été élaborées. Elles comprennent la provenance, le sexe, l'âge et l'origine suspectée de la contamination (la source d'eau de boisson, aliments et mode de vie entier des réfugiés).

\section{Traitement des données}

Les Données recueillies ont été saisies et analysées à l'aide du logiciel Excel. L'analyse statistique a utilisé le test de $\chi^{2}$ (chi2) pour la comparaison de deux variables qualitatives. Les différences ont été considérées comme significative lorsque $\mathrm{p}<$ 0,05 .

\section{Prélèvement des selles}

Les selles ont été prélevées chez les malades en provenance des différents camps des réfugiés (Amboko, Gondjé et Dosseye) pour la consultation au centre de santé de «Mbeureuh » et chez les personnes se plaignant de troubles digestifs dans les ménages du camp d' «Amboko » à Goré au Sud du Tchad.

Le camp d'Amboko qui a fait l'objet d'une enquête avec prélèvement des selles dans les ménages compte 4 zones (A, B, C et D) qui contiennent au total 21 quartiers avec une population estimée à environ 12000 personnes: zone A avec 6 quartiers (Amitié, Banga, Koukolou, Dembé, Etoile et Foukou); Zone B avec 4 quartiers (Ambiance, Bongo, Koko et Dah); zone $\mathrm{C}$ avec 5 quartiers (Allewa, Balwaye, Karko, Doso et Lafia) et 
zone D avec 6 quartiers (Ewaton, Fondo, Kissoro, Lélé, Mango et Ngassa).

Les réfugiés du camp de Gondjé et Dosseye ont été enquêtés et prélevés dans les séances de consultations médicales au centre de santé de «Mbeureuh». Les selles ont été recueillies dans de flacons stériles puis quelques grammes ont été prélevés et transférés dans un tube sec stérile de $5 \mathrm{ml}$ contenant $3 \mathrm{ml}$ de gélose Cary-Blair (CDC, 1990). Durant le séjour de l'enquête, les selles ont été conservées dans une glacière contenant de cool-box pour être transférées ensuite vers le laboratoire de l'HGRN pour des analyses.

\section{Examen de selles}

Les échantillons des selles ont été soumis à l'examen direct (microscopique) pour la mise en évidence des parasites et des levures.

\section{Culture et antibiogramme}

L'isolement et l'identification des germes ont été réalisés après ensemencement des selles sur gélose Hektoen, gélose de Saboraud chloramphénicol $\left(\right.$ Bio-Rad $\left.^{\circledR}\right)$ et gélose EMB (éosine bleu de méthylène) chez les enfants de 0 à 2 ans.

Après 18 à 24 heures d'incubation à l'étuve bactériologique à $37^{\circ} \mathrm{C}$, les colonies vertes et bleuâtres avec ou sans centre noir sur gélose Hektoen sont suspectées (Salmonella, Shigella) et celles à reflet métallique sur gélose EMB également suspectées (Escherichia coli entéropathogènes). Les colonies ont été repiquées sur gélose de Mueller-Hinton (MH) pour la coloration de Gram, le test d'oxydase et les études antigéniques. L'identification biochimique a été effectuée avec la galerie API ${ }^{\circledR} 20 \mathrm{E}$ (BioMérieux 20100). Les colonies blanches sur gélose de Saboraud chloramphénicol sont suspectes de candidose et ont été repiquées dans $1 \mathrm{ml}$ de sérum humain et placées dans l'étuve à $37^{\circ} \mathrm{C}$ pendant 24 heures pour réaliser le test de filamentation caractéristique des Candida albicans.

Le test d'agglutination a été exécuté en suivant les instructions de Kaufmann et White (Pilet et al., 1979) à l'aide des sérums : polyvalent anti-Shigella type 1, anti-flexneri, anti-boydii et anti-sonnei (Bio-Rad ${ }^{\circledR}$ ) pour la recherche des Shigella; anti-Salmonella (OMA, OMB, OMC et Vi) $\left(\mathrm{Bio}^{-\mathrm{Rad}^{\circledR}}{ }^{\circledR}\right.$ pour la recherche de Salmonella. Et pour la recherche des Escherichia coli entéropathogènes, l'agglutination a été réalisée simplement avec le sérum salin $0,9 \%$ pour éliminer les souches Rough et l'identification des pathovars d'E. coli a été effectuée par les sérums d'agglutination Nonavalents, Trivalents, Monovalents et mélange IV. La culture de l'inoculum sur la galerie $\mathrm{API}^{\circledR} 20 \mathrm{E}$ (BioMérieux 20100) a été exécutée pour l'identification biochimique. Le principe de la galerie API $^{\circledR} 20$ E est basé sur l'inoculum des microtubules avec une suspension qui réhydrate les milieux. L'incubation est à $37^{\circ}$ $\mathrm{C}$ à l'étuve pendant 24 heures au cours duquel se déroulent les réactions biochimiques (décarboxylation, fermentation, désamination) qui se traduisent par des produits colorés, spontanés, révélés par addition des réactifs. L'identification des Salmonella et des Shigella est obtenue à l'aide de catalogue $\mathrm{API}^{\circledR} 20 \mathrm{E}$. Le catalogue fournit l'identification d'un grand nombre de profil obtenu sur $\mathrm{API}^{\circledR} 20 \mathrm{E}$, ce qui confère une grande fiabilité à l'interprétation des résultats. L'inoculum McFarland 0,5 a été utilisé pour réaliser l'antibiogramme selon des techniques classiques (méthode de diffusion de disque ou technique de kirby-Bauer). La lecture des diamètres de la sensibilité des disques d'antibiotiques a été effectuée selon les recommandations du Comité de l'Antibiogramme de la Société Française de Microbiologie (CA-SFM) (CA-SFM, 2010). 
Les antibiotiques utilisés pour le test de sensibilité vis-à-vis des agents bactériens isolés étaient: Ampicilline $(10 \mu \mathrm{g})$, Amoxicilline (25 $\mu \mathrm{g})$ (Bêta-Lactamines), Ciprofloxacine $(5 \mu \mathrm{g})$ (Fluoroquinolones), Doxycycline (30 $\quad \mu \mathrm{g}) \quad$ (Cyclines), Chloramphénicol (30 $\mu \mathrm{g})$ (Phénicolés) et Cotrimoxazole $(25 \mu \mathrm{g})$ (Sulfamides) (Bio$\left.\operatorname{Rad}^{\circledR}\right)$. Ils ont été sélectionnés par rapport à leur grande prescription en milieu des réfugiés, observée lors de nos enquêtes.

Les souches ont été conservées à $-80^{\circ}$ $\mathrm{C}$ dans le bouillon cœur cervelle (BCC) (Bio$\left.\operatorname{Rad}^{\circledR}\right)$ à $15 \%$ de glycérol pour des caractérisations futures suivant les directives de National Committee on Clinical Laboratory Standards (NCCLS) de 1998 (CDC, 2002).

\section{RESULTATS ET DISCUSSION}

En guise de synthèse, il convient de répondre à la question suivante: la guerre civile au Tchad, ou d'une manière plus générale, les troubles sociaux ont-ils un impact néfaste sur la santé et l'état nutritionnel des déplacés tchadiens et les réfugiées soudanais et Centrafricains ?

La réponse à cette question nécessiterait certes la confrontation de nos résultats avec ceux d'avant la guerre civile ou les troubles sociaux, ceux d'après la guerre, cependant très peu des données actualisées existent par rapport à ce sujet.

\section{Prévalence des différents germes pathogènes isolés dans les selles en milieu des réfugiés}

Le Tableau 1 indique la répartition en nombre des cas et la prévalence des microorganismes isolés dans les selles.

Des 53 cultures effectuées, 13 agents bactériens, 10 agents parasitaires et 6 agents fongiques ont été isolés, soit 24,52\% $\left(\mathrm{x}^{2}=\right.$ $33,42, \mathrm{ddl}=1, \quad p=0,001$ différence significative $) ; 18,86 \%\left(\mathrm{x}^{2}=35,08, \mathrm{ddl}=1, p\right.$
$=0,001$ différence significative $)$ et $11,32 \%\left(\mathrm{x}^{2}\right.$ $=42,43, \mathrm{ddl}=1, p=0,001$ différence significative) respectivement. Parmi les 29 agents pathogènes isolés, il a été identifié 2 cas de co-infection bactéries/parasites, 1 cas de co-infection bactéries/agents fongiques et 2 cas de co-infection parasites/parasites. D'autres études (Reither et al., 2007) ont rapporté des cas similaires chez les enfants en milieu défavorisé au Nord du Ghana.

Par rapport à la classe d'âge, on a observé dans la tranche d'âge de 0 à 5 ans des taux élevés d'infestations des agents fongiques et des infections bactériennes de $83,53 \%$ et $61,63 \%$ respectivement. Chez les sujets de plus de 6 ans, nous n'avons notifié que le taux d'infection bactérienne $(38,46 \%)$. Par contre les taux d'infestations parasitaires étaient de $10 \%$ dans la classe d'âge de 0 à 5 ans et de plus en plus élevés chez les sujets de 6 ans et plus (Tableau 1). Les taux d'infestations (parasitaires, fongiques) et d'infections bactériennes élevés observés en milieux des réfugiés seraient directement liés à leurs conditions de vie socio-économiques et d'autre part, par la carence des aliments riches en vitamines nécessaires pour la protection et croissance des enfants en milieux confinés. En outre, le mauvais assainissement est un facteur contributif, de par son impact sur les taux de malnutrition, aux autres causes principales de mortalité infantile, notamment le paludisme, les maladies respiratoires graves et la rougeole (ACF/FR, 2007).

Il ressort de ces résultats que, en milieu des réfugiés en dehors des protozoaires, les principales causes des troubles digestifs sont les bactéries (Escherichia coli, Salmonella et les Shigella). Ces résultats montrent que les agents bactériens impliqués dans les maladies diarrhéiques seraient liés à l'alimentation des refugiés. La mise en cause des infections bactériennes dans les maladies diarrhéiques 
par le mode de l'alimentation a été également rapportée à Ouagadougou (Barro et al., 2007).

En plus des maladies diarrhéiques, l'enquête a permis de relever en milieux des refugiés des deux camps d'autres cas de maladies dans 205 ménages : 106 (51,70\%) cas de paludisme, $23(11,21 \%)$ cas de VIH/SIDA et $76(37,07 \%)$ cas d'autres affections (Otites, dermatoses, angines, gales, rhume rougeole, rhinites, carie dentaire et les hépatites). Des résultats similaires ont été également rapportés dans 12 camps des réfugiés à l'Est : le paludisme avec 43889 cas dont 65 décès soit une létalité de $0,14 \%$; Hépatite E dans le district sanitaire de GozBeida avec 3359 cas dont 45 décès soit un taux de létalité de $1,33 \%$ et les infections respiratoires avec 1224976 cas dont 68 décès soit un taux de létalité de 0,05\% (OMS, 2008 ; Giuliano et al., 2008). Les cas de certaines maladies telles que: gales, dermatoses, typhoïdes, parasitoses seraient attribuables à la malnutrition. Par ailleurs, Chahed et al. (2007) soulignent que certains Escherichia coli producteurs de Shigatoxines (STEC) sont responsables de toxi-infections d'origine alimentaire qui se traduisent par des diarrhées mais aussi par des syndromes plus graves pour l'homme comme le syndrome hémolytique urémique pouvant provoquer la mort.

\section{Étude de la sensibilité des agents bactériens aux antibiotiques}

Le Tableau 2 montre l'évaluation de l'efficacité des antibiotiques vis-à-vis des agents bactériens isolés. L'étude a concerné 6 antibiotiques testés sur les agents bactériens isolés : 4 Escherichia coli, 6 Shigella flexneri, 2 Salmonella Typhi et 1 Salmonella paraTypi A. Les proportions de résistance (R), intermédiaire (I) et sensible (S) ont été obtenues.

La plupart des souches isolées ont été résistantes aux bêta-Lactamines
(Ampicilline, Amoxicilline) soit une proportion d'environ $85 \%$. Cette augmentation de la résistance serait due à la prescription abusive et inappropriée par des agents de santé que l'on a observée pendant notre enquête. La résistance croisée a été également observée dans cette famille par la résistance à $13(100 \%)$ de l'utilisation abusive de l'ampicilline, qui a entraîné une résistance croisée de $10 \quad(77 \%)$ à l'amoxicilline et 3 (23\%) d'intermédiaire. Une telle résistance lorsqu'elle est observée, serait liée à la production de bêta-lactamases de types pénicillinases par la souche en question (Zeba et al., 2007; Hauka et Siitonen, 2007). Nos résultats peuvent s'expliquer par ce phénomène. La résistance des antibiotiques tels Cotrimoxazole 6 (46\%), Doxycycline 12 (92\%) et Chloramphénicol 6 (46\%) pourrait également s'expliquer par la prescription inappropriée de ces antibiotiques par les agents de la santé dans les centres de santé en milieux des réfugiés sans évidence de laboratoire. Par contre, elles ont été sensibles à 98\% des Fluoroquinolones (Ciprofoxacine). Cette sensibilité a été également observée par Karki et Tiwari (2007) dans la vallée de Kathmandu au Népal.

\section{Fréquence de consommation d'aliments dans le ménage en milieux des réfugiés au Tchad}

Le Tableau 3 indique le nombre des repas consommés dans chaque ménage en milieux des réfugiés à Goré au Sud dans le camp d'Amboko et à GozAmir (GozBeida) à l'Est du Tchad.

$$
\text { L'enquête au camp d'Amboko }
$$
à Goré au Sud a permis de relever sur 107 ménages: 0 repas $5(4,6 \%), 1$ repas 53 $(49,5 \%), 2$ repas $37(34,5 \%), 3$ repas 12 $(11,2 \%)$ et 3 repas et plus $0(0 \%)$ et celle du camp de GozAmir de Gozbeida à l'Est sur 98 ménages: 0 repas $3(3,0 \%), 1$ repas 13 
$(13,2 \%), 2$ repas $58(59,1 \%), 3$ repas 23 $(23,4 \%)$ et 3 repas et plus $1(1 \%)$. Le nombre de repas qui variait généralement de 1 à $2 /$ jour et rarement de 3/jour dans les deux camps ne pourrait pas permettre de couvrir qualitativement le besoin nutritionnel (2100 Kcal) de chaque refugié/jour prévus par le Haut Commissariat des Nations Unies pour les réfugiés (UNCHR) et le Programme Alimentaire Mondial (PAM). D'après l'Organisation Internationale des Normes (NF en ISO 9000, 2005), la qualité est l'aptitude d'un ensemble de caractéristiques intrinsèques à satisfaire des exigences. Nos résultats de 3 repas/jour $(11,2 \%)$ dans la zone des refugiés au Sud et de 3 repas/jour $(23,4 \%)$ dans la zone des réfugiés à l'Est étaient moins que ceux de HCR qui a prévu pour 2011, la consommation de $25 \%$ de 3 repas/jour de chaque ménage de refugiés soudanais et de $50 \%$ pour les réfugiés centrafricains au Sud. A cela s'ajoute les proportions sur les différents logements que l'enquête a relevé dans les deux camps HCR : tente en bâche 67 (32,68\%), hangar 89 $(43,14 \%)$ et autres (cases en terre battue, en banco, en bambou) 49 (23,90\%). L'enquête a également relevé les types de logements : natte et matelas par terre $152(74,14 \%)$, lit et matelas $6(2,92 \%)$ et autres (natte par terre, lit traditionnel sans matelas) 47 (22,92\%). Nos résultats relatifs aux logements et types de logements sont en dessous de la projection de HCR qui avait prévu pour 2011, une amélioration qualitative des logements adéquats à tous les réfugiés du Sud du Tchad. Les résultats de types de logement associés au manque d'hygiène des ustensiles utilisés pour la préparation des aliments, suivis de l'insuffisance de l'hygiène corporelle et vestimentaire n'est pas en conformité avec les affirmations de HCR/PAM (2008) qui stipulent que l'état de la sécurité alimentaire d'un foyer de réfugiés ou de personnes déplacées à l'intérieur du pays ou d'un individu est généralement déterminé par l'interaction d'un large éventail de facteurs agro-environnementaux, socioéconomiques et biologiques. Les types de latrines observés pendant les enquêtes étaient à $100 \%$ traditionnels et le plus souvent à visage découvert. Dans les deux camps des refugiés (Est et Sud) enquêtés, le ratio utilisateurs/latrine était de 36 et 33 refugiés/latrine, les standards étaient fixés à 20 personnes/latrine (HCR/PAM, 2008). Ce faible ratio pourrait s'expliquer par une forte participation des bénéficiaires et serait à l'origine de la contamination des aliments par des agents vecteurs de maladies (mouches, cafards...) et par conséquent des maladies diarrhéiques en milieux confinés (OMS, 2008). D'autres études (De Jesus et al., 2004 ; Szalanski et al., 2004) ont montré que les insectes jouent un rôle important dans la transmission des microorganismes pathogènes responsables des infections. La prise alimentaire inadéquate serait à l'origine des maladies diarrhéiques chroniques, hypoglycémie, retard de croissance et autres pathologies digestives (cirrhose biliaire ou éthylique, pancréatite chronique recto-colite hémorragique) observés pendant nos enquêtes.

Les aliments les plus associés aux troubles digestifs en milieux des réfugiés au Tchad

Le Tableau 4 montre les groupes d'aliments les plus impliqués dans les troubles digestifs en milieux des refugiés au Tchad. A Amboko à Goré au Sud dans la zone forestière et humide, les aliments les plus fréquemment consommés sont les légumes $18,6 \%$ suivie des céréales 22,8\% par contre à GozAmir à l'Est dans le désert, la fréquence de la consommation des céréales est prédominante, soit 33,5\% comparée aux légumes 5,6\%. Les produits laitiers, les boissons locales et les eaux de consommations sont les aliments sensibles et très souvent ils sont contaminés 
au cours de leur préparation par les mains sales. C'est ce qui explique leur fréquence dans les troubles digestifs. Barro et al. (2005) ont montré que les céréales et les sauces de légumes sont généralement consommées en partie et le reste est conservé pour un second repas au coucher dans des conditions d'hygiènes précaires les exposant à la contamination microbienne et physique. Ces aliments déjà préparés ne peuvent pas être préparés à nouveau, on peut les réchauffer, ce qui n'élimine pas tous les germes. La consommation insuffisante des aliments constatée en milieux des réfugiés ne pourrait sécuriser leur régime alimentaire si l'on considère comme l'avait prévu le sommet mondial de 1996 sur l'alimentation (FAO, 2006) qui indique que la sécurité alimentaire existe lorsque les êtres humains ont, à tout moment, un accès physique social et économique à une nourriture suffisante, saine et nutritive leur permettant de satisfaire leurs besoins énergétiques et leurs préférences alimentaires pour mener une vie saine et active. Les boissons locales après la préparation sont vendues pendant de longues heures les exposant à la contamination par des mouches. D'après Allan et Zurek (2004), l'espèce de mouche Musca domestica est reconnue comme vecteur et responsable de plusieurs maladies dans tous les continents. Par rapport à la consommation d'eau dans l'Est du Tchad, la fourniture d'eau aux refugiés est de 12 à 15 litres/personne/jour, laquelle est beaucoup moins que celle recommandée par HCR/PAM (2008) qui est de 20 litres/personne/jour. Dans certaines localités des réfugiés, l'enquête a relevé le non fonctionnement des pompes d'eau de forage par manque des pièces de rechange. Cette situation de panne mécanique ramène la fourniture d'eau à 6 litres/personne/jour aux réfugiés les exposant à la déshydratation et même serait à l'origine des risques de contamination fécale par des eaux des puits et mares consommées pendant la période de pénurie.

Tableau 1: répartition des germes pathogènes selon la tranche d'âge.

\begin{tabular}{lccccc}
\hline Microorganismes & \multicolumn{5}{c}{ Tranche d'âge } \\
\cline { 2 - 6 } & $\mathbf{0 - 5}$ ans & $\mathbf{6 - 1 0}$ ans & $\mathbf{1 1 - 1 5}$ ans & $\mathbf{1 6 - 2 0}$ ans & $\mathbf{2 1}$ ans et + \\
\hline Protozoaires & 1 & 1 & 2 & - & 2 \\
Entamoeba histolytica & - & - & - & - & 2 \\
Giardia intestinalis & - & - & - & - & 2 \\
Trichomonas intestinalis & $1(10)$ & $1(10)$ & $2(20)$ & - & $6(60)$ \\
Total (\%) & & & & - & - \\
\hline Agents bactériens & 4 & - & - & - & 4 \\
Escherichia coli & 2 & - & - & - & 1 \\
Shigella flexneri & 1 & - & - & - & - \\
Salmonella Typhi & 1 & - & - & - & $5(38,46)$ \\
Salmonella para Typhi A & $8(61,63)$ & - & - & - & - \\
Total (\%) & & & & \\
\hline Agents fongiques & $5(83,53)$ & - & $1(16,66)$ & - & - \\
Candida albicans & &
\end{tabular}


Tableau 2: Efficacité des antibiotiques.

\begin{tabular}{|c|c|c|c|c|c|c|c|c|c|c|c|c|c|c|c|c|c|c|c|}
\hline \multirow{3}{*}{ Agents bactériens } & \multirow{3}{*}{$\begin{array}{l}\text { Nbre } \\
\text { testé }\end{array}$} & \multicolumn{18}{|c|}{ Antibiotiques testés } \\
\hline & & \multicolumn{3}{|c|}{ Ampicilline } & \multicolumn{3}{|c|}{ Amoxicilline } & \multicolumn{3}{|c|}{ Doxycycline } & \multicolumn{3}{|c|}{ Chloramphénicol } & \multicolumn{3}{|c|}{ Ciprofloxacine } & \multicolumn{3}{|c|}{ Cotrimoxazole } \\
\hline & & $\mathbf{R}$ & I & $\mathbf{S}$ & $\mathbf{R}$ & $\mathbf{I}$ & $\mathbf{S}$ & $\mathbf{R}$ & $\mathbf{I}$ & $\mathbf{S}$ & $\mathbf{R}$ & I & $\mathbf{S}$ & $\mathbf{R}$ & $\mathbf{I}$ & $\mathbf{S}$ & $\mathbf{R}$ & I & $\mathbf{S}$ \\
\hline$\overline{\text { Escherichia coli }}$ & 4 & 4 & 0 & 0 & 4 & 0 & 0 & 4 & 0 & 0 & 2 & 1 & 1 & 0 & 1 & 3 & 4 & 0 & 0 \\
\hline Shigella flexneri & 6 & 6 & 0 & 0 & 4 & 2 & 0 & 5 & 1 & 0 & 4 & 1 & 1 & 0 & 1 & 5 & 2 & 1 & 3 \\
\hline Salmonella Typhi & 2 & 2 & 0 & 0 & 2 & 0 & 0 & 2 & 0 & 0 & 0 & 1 & 1 & 0 & 0 & 2 & & 0 & 2 \\
\hline Salmonella para Typhi A & 1 & 1 & 0 & 0 & 0 & 1 & 0 & 1 & 0 & 0 & 0 & 0 & 1 & 0 & 0 & 1 & 0 & 0 & 1 \\
\hline Total & 13 & 13 & $\mathbf{0}$ & $\mathbf{0}$ & 10 & 3 & $\mathbf{0}$ & 12 & 1 & $\mathbf{0}$ & 6 & 3 & 4 & $\mathbf{0}$ & 2 & 11 & 6 & 1 & 6 \\
\hline
\end{tabular}

Tableau 3 : Fréquence de repas dans chaque ménage en milieux de réfugiés au Tchad.

\begin{tabular}{ccccccc}
\hline Camps HCR & \multicolumn{5}{c}{ Nombre de repas } & Total \\
\cline { 2 - 6 } & 0 repas & 1 repas & 2 repas & 3repas & 3 repas et + & \\
\hline Amboko & 5 & 53 & 37 & 12 & 0 & 107 \\
Proportions des repas (\%) & $4,6 \%$ & $49,5 \%$ & $34,5 \%$ & $11,2 \%$ & $0 \%$ & \\
GozAmir & 3 & 13 & 58 & 23 & 1 & 98 \\
Proportions des repas (\%) & $3,0 \%$ & $13,2 \%$ & $59,1 \%$ & 23,4 & $1,0 \%$ & \\
\hline
\end{tabular}


Tableau 4: Évaluation de la fréquence des différents groupes d'aliments dans les troubles digestifs en milieux des réfugiés au Tchad.

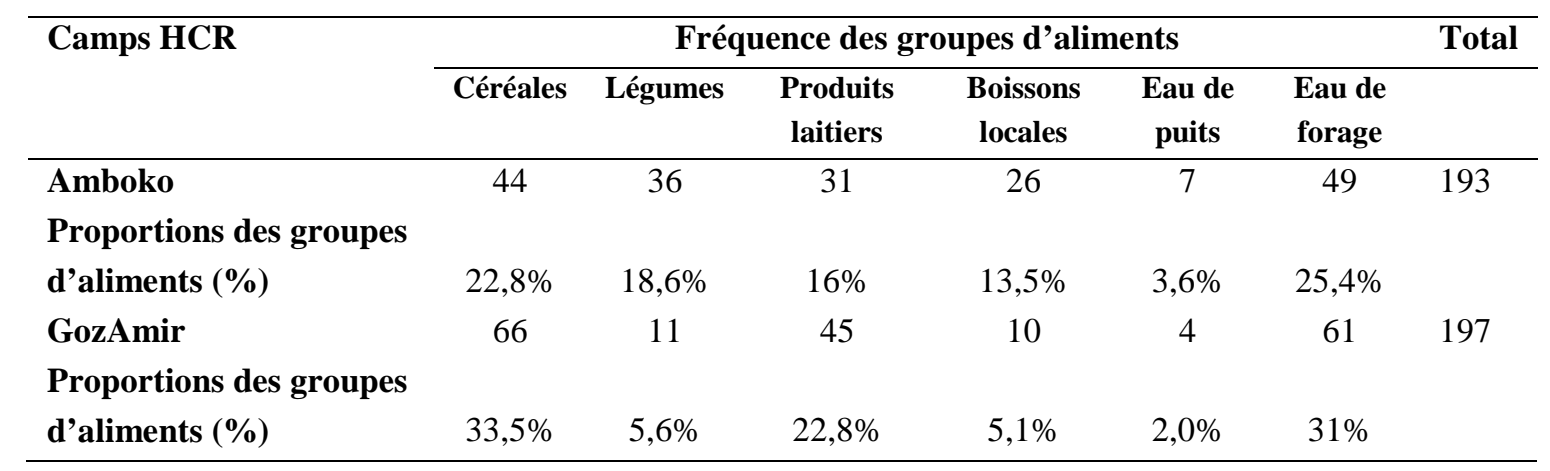

La contamination fécale des eaux des puits mal protégés, des rivières et des marres a été également rapportée par Barro et al. (2005). Nos enquêtes ont également relevé les difficultés d'accès à la nourriture dans les camps des réfugiés. Les aides alimentaires accordées par le HCR aux réfugiés sont constituées de sucres, céréales, biscuits et huiles qui ne sont plus que de source énergétique. Ce qui ne permettrait pas aux réfugiés de couvrir leurs besoins alimentaires tel que stipulés par le HCR/PAM (2008) comme quoi, l'accès à la nourriture est la capacité d'un foyer d'obtenir régulièrement des qualités de nourritures adéquates en associant ses propres réserves et sa production personnelle, ses achats, trocs, cadeaux, emprunts ou son aide alimentaire. Ces aliments à haute valeur énergétique seraient à l'origine des cas de dénutritions mixtes du fait de leur catabolisme et de carence d'apport en oligo-éléments et qui traduirait la typologie clinique de diabète insulinodépendant et des œdèmes dues à la protéinurie observée chez certains réfugiés pendant nos enquêtes.

\section{Conclusion}

Les réfugiés sont des groupes de personnes vulnérables dans un environnement confiné. Ils sont victimes des maladies infectieuses causées par les Salmonella, Shigella, et Escherichia coli entéropathogénes qui représentent $24,52 \%$ de tous les agents pathogènes impliqués dans les maladies diarrhéiques montrant ainsi un problème de santé publique que causent ces bactéries en milieu des réfugiés au Tchad. Ces maladies diarrhéiques à entéropathogènes sont dues à la situation générale des infections liées aux conditions socio-économiques des réfugiés. En plus, les souches isolées ont été résistantes à $85 \%$ aux antibiotiques de la famille des bêta-Lactamines, compliquant davantage la situation. En outre, cette étude a permis de mettre en exergue la prévalence et la fréquence des entéropathogènes dans les selles en milieu des réfugiés au Tchad liées à la malnutrition et au manque d'hygiènes, généralement, associés aux maladies diarrhéiques. Le contrôle de ces infections requiert un effort concerté entre les différents acteurs (gouvernements, les services de base des humanitaires et les réfugiés). D'autre part, $51,70 \%$ de cas de paludisme et $11,21 \%$ de VIH/SIDA ont été enregistrés. Par rapport à la situation nutritionnelle, l'enquête a permis de relever que le nombre de repas consommé en milieu des réfugiés est insuffisant pour couvrir leurs besoins alimentaires quotidiens. 


\section{REMERCIEMENTS}

Les auteurs remercient les coordonnateurs du Centre de Support en Santé Internationale des deux camps (Est et Sud) des réfugiés au Tchad pour avoir contribué à cette étude.

\section{REFERENCES}

Allan M.J, Zurek L. 2004. Association of Escherichia coli with houseflies on a cattle farm. Appl. West Afr. Food Nutr., 3: 7578-7580.

ACF/FR (Action Contre la Faim/France). 2007. Dynamique des interactions : Malnutrition, eau, assainissement hygiène, infection, $7 \mathrm{p}$.

Barro N, Sangaré L, Tahita M-C, Ouattara C A T, Traoré AS. 2005. Les principaux agents du péril fécal identifié dans les aliments de rue et ceux des cantines et leur prévalence en milieu hospitalier: Séminaire régional scientifiques et pédagogiques : «Maîtrise » des procédés en vue d'améliorer la qualité et la sécurité des aliments, l'utilisation des OMG, analyse des risques en agroalimentaire. Du 8 au 11 Novembre 2005, CIDEFA, Ouagadougou, Burkina Faso, 113-118.

Barro N, Gamene AA, Itsiembou Y, Savadogo A, Nikiema AP, Outtara CAT, de SOUZA CA, Traore AS. 2007. Streetvended Food improvement: Contamination Mecanism and Application of Food Safety Objective Strategy: Critical review, Pakistan Journal of Nutrition, 6(1): 1-10

Breiman RF. 2009. Cholera outbreak in Kenyan refugee camp: risk factors illness and importance of sanitation. The American Society of Tropical Medicine and Hygiene, 80(4): 640-645.

CDC (Center for Disease Control and Prevention). 1990. Recommendations for the collection of laboratory specimens associated with outbreaks of gastroenteritis. MMWR., 39(14): 14-16.

CDC (Centre for Diseases Control And Prevention). 2002. Méthodes de Laboratoires pour le Diagnostic de la Dysenterie Epidémique et du Choléra. Centre for Diseases Control And Prevention : Atlanta Georgia. 172p.

Chahed A, China B, Daube G. 2007. Les Escherichia coli producteurs de Shigatoxines dans les toxi-infections d'origine alimentaire. Ann. Méd. Vét., 151: 215-224.

CA-SFM (Comité d'Antibiogramme de la Société Française de Microbiologie). 2010. Recommendations 2010.

De Jesus AJ, Olsen AR, Bryce JR, Whiting RC. 2004. Quantitative contamination and transfer of Escherichia coli from foods by houseflies, Musca domestica L. (Dipteria: Muscidae). In. J. Food Microbiol., 93 : 259-262.

FAO. 2006. Sécurité alimentaire. Note d'orientation $\mathrm{N}^{\circ}$ 2, FAO, 4 p.

Giuliano M, Walter C, Djaousede, Madjiangar P, Naissem J, Mbaitoubam T, Katir N, Rehr A. 2008. Faits et chiffres point de situation, 29 Juillet 2008. Bull. Info. UNHAS, 1-12.

Haukka K, Siitonen. 2007. Emerging resistance to never and antimicrobial agents among Shigella isolated from finish foreign travellers. Epidemiol Infect., 136: 476-482.

HCR/PAM. 2008. Directive pour Mission d'Evaluation Conjointe HCR/PAM $\left(2^{\text {ème }}\right.$ édn). Haut Commissariat des Nations Unies pour les Réfugiés et Programme Alimentaire Mondial, HCR/PAM; 265 p.

IMC (International Medical Corps). 2010. Exercice de cartographie. Rapport de IMC, 11-13.

Karki A, Tiwari Br. 2007. Prevalence of Acute Diarrhoea in Kathmandu Valley. 
Journal Nepal Medecin Associate, 46(4): 175-179.

Mockenhaupt FP. 2007. Acute childhood diarrhoea in nother Ghana: Epidemiological, Clinical and Microbiological characteristics. BMC Infectious Diseases, 104(7) : 8.

NF en ISO 9000. 2005. Lexiques définitions normalisées (ISO 9000). Extrait du document de l'Association Française de Normalisation (AFNOR).

OMS. 2008. Choléra-Zimbabwé. REH., 83: 449-450.

Pilet C, Bourdon JL, Toma B, Marchal N, Balbastre C. 1979. Bactériologie Médicale et Vétérinaire: Systématique Bactérienne ( $2^{\text {ème }}$ edn). DOIN Editeur : Paris, France; 437p.
Szalanski AL, Owens CB, McKay T, Steelman CD. 2004. Detection of Campylobacter and Escherichia coli 0157:H7 from filth flies by polymerase Chain reaction. Med. Vet. Entomol., 18: 241-246.

WSP (Water and Sanitation Program/Tchad). 2012. Impacts économique d'un mauvais assainissement en Afrique. Document WSP, $6 \mathrm{p}$.

Zeba B, Kiendrebeogo M, Lamien A, Docquier JD, Simporé J, Nacoulma GO. 2007. Major enzymatic Factors involved in Bacterial Penicillin Resistance in Burkina Faso. Journal of Biological Sciences., 10(3): 506-510. 